\title{
Recorridos, tensiones y desplazamientos en el ideario de Alicia Moreau ${ }^{1}$
}

\section{Paths, tenseness and tension displacements in Alicia Moreau's ideas}

\author{
Adriana María Valobra \\ CINIG/IDIHCS - UNLP/CONICET, Argentina \\ indivalobra@gmail.com
}

\begin{abstract}
SÍNTESIS
Este estudio es, en primer lugar, un ejercicio de visibilización de la obra de Alicia Moreau de Justo -una de las figuras centrales del feminismo sufragista argentinoy un primer paso para problematizar la relación entre su ideario, la coyuntura y los procesos históricos que le tocaron vivir. Nos proponemos analizar su producción profesional y política señalando cómo va modelando sus miradas sobre las mujeres y la acción política. Al mismo tiempo, y en estrecha vinculación a lo anterior, ella construye una propuesta democratizadora que se tensará con la llegada de Juan Domingo Perón al gobierno en 1946 y aún después de que éste fue derrocado.
\end{abstract}

\begin{abstract}
This study aims to make visible one of the most important feminist and sufraggettet in Argentina. Alicia Moreau de Justo. We will focuses on her work in order to problematize the connection between her ideas, the junctures and historical processes she lived through. The study analyzes her professional and political interventions highlighting her views about women and political action. We will focus on Alicia's sight about women and political action. Besides, and closely tied with those ideas, she builds a democratizing proposal. But, Moreau's ideas were affected by the arrival of Juan Domingo Perón arrived to the Argentine government (1946) and even after it was overthrown.
\end{abstract}

Palabras claves: Alicia Moreau de Justo, trayectoria intelectual, ideario político, crítica científica, desde 1906 hasta 1957.

Keywords: Alicia Moreau de Justo, intellectual trajectory, political ideology, scientific criticism, from 1906 to 1957. 
Una frondosa producción reciente sobre la historia intelectual y de los intelectuales ha dejado un injusto vacío en torno de una figura central del pensamiento político argentino: Alicia Moreau de Justo, una de las referentes del feminismo, sufragismo y socialismo en Argentina ${ }^{2}$. Algunos encuentran que es reñido incluir a esta figura en el horizonte intelectual debido a su militancia partidaria en el socialismo. La noción de intelectual orgánico se utiliza para denominar casos semejantes, aunque muchas veces aleja del universo intelectual (Terán, 1993). Sin embargo, poco se ha escrito sobre las diferencias que Alicia expresó en su producción respecto de las líneas más hegemónicas del partido o, en el mejor de los casos, apenas se ha enunciado ese pensamiento diferencial (Tortti, 2009). Su obra relacionada a la educación y al sufragio femenino ha sido, para algunas autoras, más conocida (Lavrin, 1997; Barrancos, 1996). Como ha señalado Dora Barrancos, se ha facilitado su expresión como "educacionista, pero no como 'cientista'" (1996: 226).

Considero que este silencio se debe a que Alicia se ajusta poco a cualquier catalogación que haya sido pensada en términos masculinos. En efecto, aún con la privacidad que ella mantuvo sobre su vida personal, el conocimiento de que colocaba un cartel en la puerta de su escritorio que decía "No molestar, mamá trabajando", posiciona la cuestión de lo público y lo privado en una difícil tensión que se ha resuelto, la más de las veces, omitiéndola. El hecho de que su vasta producción intelectual no haya merecido una reflexión más allá de lo biográfico y que aún se encuentre dispersa, puede resultar un indicador claro de esa desconsideración.

Este estudio es, en primer lugar, un ejercicio de visibilización de su obra y un primer paso para problematizar la relación entre su ideario, la coyuntura y los procesos históricos de más lenta maceración que le tocaron vivir. Intentamos franquear cualquier intento de "ilusión biográfica" pues, junto con Bourdieu (1997), no buscamos la coherencia del ideario de Alicia Moreau de Justo, sino que señalaremos las torsiones que fueron sufriendo sus pensamientos, a la luz de contextos cambiantes que, en algunos momentos, acicatearon esos cambios y, en otros, estancaron su fluir. Nos proponemos analizar su producción profesional y política señalando cómo va modelando sus miradas sobre las mujeres y su acción política reconstruyendo su biografía intelectual a la vez que esbozando elementos del estado 
del campo intelectual y político (Bourdieu, 2002: 104). Las etapas de su ideario, en realidad, no lo son en el sentido consecutivo; sino por el predominio de algunas preocupaciones más que otras en relación con su producción escrita que es la que utilizaremos.

Nuestra hipótesis es que, tempranamente, Alicia Moreau consideró a los sujetos sociales con un mínimo componente biológico, apenas un sustrato. La importancia que ella otorgó al medio social como modelador de la subjetividad estuvo estrechamente vinculada con sus ideas respecto de los roles sociales de las mujeres y su papel en el mundo de lo político. Consideraba que las mujeres debían fortalecer y perfeccionar la democracia, cuya simiente estaba en la cultura argentina. La llegada de Juan Domingo Perón al gobierno argentino (1946) conmovió sus ideas respecto de la existencia de esa raíz democrática. Sin embargo, durante la "Revolución Libertadora" que terminó de facto ese gobierno, la actitud antiperonista de Moreau entrará en conflicto con sus postulados pacifistas y ello evidenciará una tensión en su propio discurso, aun cuando intentó retomar aquellas ideas sobre el rol de la educación en la formación del ciudadano y el papel preponderante que tenían las mujeres.

\section{Lo biológico en cuestión}

A comienzos del siglo XX, las teorías imperantes invocaban a sofisticadas fórmulas (que hoy consideraríamos seudo científicas, aunque entonces integraron el sustrato indiscutible de la ciencia) que mixturaban elementos de la frenología, la craneometría y la biotipología con el objetivo de encontrar alguna explicación que diera sustento objetivo, observable y numéricamente demostrable para lograr comprobar que las mujeres tenían una inferioridad biológica constitutiva que hacía incompatibles las funciones maternas con la realización de actividades científicas e intelectuales (Gómez Rodríguez 2005: 478-492). Alicia Moreau fue una de las pioneras en la Facultad de Medicina de la UBA buscando desafiar esas ideas (Ramacciotti y Valobra, 2011). Tanto en esos claustros como en cursos que tomó luego de graduarse como maestra, produjo varios artículos vinculados al quehacer científico. Según la historiadora Dora Barrancos, las numerosas conferencias de Alicia "relacionadas con la medicina y la higiene social, tanto como otros abordajes próximos, 
no obtienen una edición que nos permita acceder a sus ideas en la materia" (1996: 226).

En este apartado nos enfocaremos en sus escritos de tipo médico-psicológicos en los que una Alicia joven, enérgica y aguda, aborda muchas y disímiles temáticas propias, además, de un momento formativo. Los escritos incluyen temas darwinianos y de la evolución en general, educación y temáticas ginecológicas, entre otros aspectos.

Las intervenciones médicas en el campo psicológico se habían ampliado a principios de siglo pues, así como el médico podía curar el cuerpo humano, también podía operar sobre el social. De allí que-positivismo comtiano mediante, aunque no menos influido por Claude Bernard- la razón experimental fue la base para observar y comprender lo social, incluyendo las patologías propias de una sociedad en conflicto. Así, la locura y la criminalidad fueron tratadas desde el campo médico y se sostuvo que sanar el cuerpo social requería metodologías específicas de intervención que permitieran registrar hechos observables de manera objetiva para, luego, tabular y sistematizar los resultados (Vezzetti, 1991; Huertas, 1991). Las primeras obras de Moreau se encuentran en relación con la psicología. Un primer trabajo fue compilado por la cátedra de Horacio G. Piñero (1869-1919), destacado médico psiquiatra argentino, una obra que contenía los estudios realizados en el marco del laboratorio de Psicofisiología. Alicia se basó en una entrevista que había realizado a una interna del Hospicio Nacional de Alienadas. En ella sigue con rigurosidad la metodología de indagación vigente -propia del método psiquiátrico- en la cual aguza la sospecha de que la paciente miente y que, parte de la cura, está en reconocer sus contradicciones. $\mathrm{Al}$ contrario de las tipologías del campo psiquiátrico de la época que establecen correlaciones físico-mentales, el informe de Alicia ofrece un registro detallado en el que afirma que esa patología mental diagnosticada no se corresponde con ninguna evidencia física e, incluso, hace hincapié en que la formación general de la paciente no ha sido afectada y es locuaz, con buena dicción y cierta cultura general. A tal punto, señala, que podría dudarse de que la enferma lo es (Moreau, s/d a).

A este primer trabajo, sucedió otro en coautoría con un compañero de estudios, Pedro Fernández, en el año 1905 (Moreau y Fernández, 1910). Esta investigación de tipo psicométrica, rama propia de 
la psicología experimental impulsada por Víctor Mercante, se refería a un grupo de escolares organizados por edad y por sexo (Ascolani, 2008). Según afirman, ante un excitante, la capacidad asociativa e interpretativa de los escolares era mayor que con dos. Si bien utilizaban el método psicométrico, Moreau y Fernández creían que con éste no se obtenían conclusiones sobre desarrollo intelectual infantil $\mathrm{y}$, a priori, sostenían que el comportamiento del sujeto o la manera de contestar eran también relevantes y que los resultados de su experimentación debían situarse en ese marco más amplio.

En un artículo de 1906, a instancias de su experiencia en la cátedra de Piñero, la autora defendía la psicología experimental como una vertiente que no anteponía teorías previas sino que se rendía ante los hechos. Según Moreau, esta rama no se ocupaba ya de buscar la psiquis en los primeros fenómenos biológicos siguiendo su derrotero a lo largo de la evolución, sino que buscaba "el despertar de los fenómenos psíquicos en ese compendio de la evolución de las especies que era el niño". Señalaba la necesidad de que la pedagogía ingresara decididamente al universo de las disciplinas experimentales pues consideraba que, al ser la educación una de las influencias primordiales sobre la modelación del individuo, era perentorio que aplicara un método científico. Concluía con una esperanza y una certeza a partir de ello: a futuro, "ya no se inculcarán en el niño desde muy pequeño, esas fórmulas, esas sentencias en las cuales debe creer por sólo la autoridad del que lo dice y se le acostumbrará en la familia y en la escuela a observar y razonar, se formarán en él hábitos intelectuales más positivos que lo alejarán de la palabrería hueca y sentenciosa" (Moreau, 1906: 258). Imbuida de expectativa en la cientificidad futura de la pedagogía, apostó fuertemente al papel del medio sobre cualquier herencia biológica (Talak, 2010). Estas ideas las desarrolló en la Revista Socialista Internacional que analizaremos en el siguiente apartado.

Como mencionan sus biógrafas (Cichero, 1994 y Henault, 1983), Alicia Moreau de Justo fue lectora de Charles Darwin y Herbert Spencer, obras de lectura habitual en la época, que además recibió de su padre y algunos docentes de la Escuela Normal. La influencia de esos autores, como indica Álvaro Girón (2005), requiere precisiones geográficas, temporales y culturales, su recepción y apropiación pues, en muchos casos, más que frente a adopciones irrestrictas, nos 
encontramos, más bien, con aceptaciones de la idea general de evolución antes que de las teorías y mecanismos que involucraban esas posiciones. En 1915, adhirió a la idea de que hasta los organismos denominados inferiores tenían inteligencia en tanto eran capaces de adaptar su conducta a fin de sobrevivir o mejorar sus condiciones de vida. Junto con él, también concibió que con sensibilidad, deseo y frustración se avanzaba en el orden de la naturaleza desde los protozoos hasta los seres humanos. Sin embargo, no apostaba a una psicología antropomórfica lineal que era una visión simplificada de la teoría de Darwin (Moreau, 1908: 253). Luego, según la autora, sobrevino una reacción a esta postura y fue una visión antagónica de aquella que concibió todo de manera mecánica y que mostraba al individuo obrando bajo las fuerzas externas de la naturaleza independientemente de la fisiología. Censuraba, así, ambas posturas extremas y, aunque asumía las ideas evolucionistas, lo hacía con menos ahínco en los giros biologicistas comúnmente asociados a ellas ${ }^{3}$. Esa idea se visibilizó, también, en la Conferencia "La pretendida degeneración de las razas" dictada en la Universidad Nacional de La Plata en 1907 y publicada en 1909. Es una interesante interpretación sobre el concepto de raza y las potencialidades de desenvolvimiento de los seres humanos en un medio propicio y con las herramientas educacionales adecuadas. En este texto, impugnó “el dogma prejuicioso de la pureza, la desigualdad y la supremacía raciales" (Biagini, 2004: 165). Siguiendo la tesis de Jean Finot, pensador francés que discutía las teorías racistas, Alicia utilizó el concepto para dar cuenta de aspectos culturales más que de los biológicos.

En 1914 se publicó su tesis de grado, apadrinada por el Dr. Jaime Salvador, un médico interno del Hospital Nacional de Clínicas -primero en lograr en Argentina un "injerto" exitoso de ovario-, se denominó “La función endócrina del ovario" y se enmarcó en lo que podemos considerar una temática aceptada por los cánones de la época para una mujer, la ginecología, aunque -a su vez- ello implicó desplazar a los varones que se habían autoerigido como representantes de un saber sobre el cuerpo de las mujeres (Nari 2004). Según afirmaba la tesista, el estudio fue sugerido por las enfermeras del Hospital en el que realizó su residencia, un reconocimiento que evidenciaba la sororidad para con este cuerpo de reciente trayectoria en el país y cuya formación profesional había encarado la doctora 
Cecilia Grierson. Su estudió se inscribió en un campo relativamente nuevo de la medicina: la ginecología endócrina (Aparicio, 2006). Las investigaciones eran aún incipientes cuando Alicia escribió su tesis y se enfocó en demostrar la existencia de la secreción ovárica y la función endócrina sobre los órganos genitales y el resto del organismo $\mathrm{y}$, fundamentalmente, reflexionar sobre el papel del cuerpo amarillo en la preparación y mantenimiento del útero para el embarazo así como la influencia en distintas enfermedades. Moreau recogió exhaustivamente las novedades en la materia y tomó una distancia crítica respecto de los métodos y técnicas de investigación sobre las cuales se hacían conclusiones. Entre otras consideraciones, señalaba que uno de los problemas que conspiraban contra la recopilación documental y la casuística era la falta de formación de los colegas en el método experimental ${ }^{4}$. Asimismo, sostenía: "no puede concluirse de los resultados obtenidos en otras especies animales sólo basándose en analogías" (Moreau, 1914: 204).

Respecto de las consideraciones sobre el cuerpo lúteo, masa que aparece en el ovario después de la ovulación y que se consideraba el que, mediante producción de hormonas, facilitaba la gestación. Moreau afirmó, "si en las especies animales estudiadas parece evidente que la presencia del cuerpo amarillo es necesaria para la gestación, no está demostrado que igual cosa suceda en las especie humana" (1914: 72). A partir de allí, la tesista aportaba algunas lecturas que mostraban que el intento de establecer correlaciones entre el ciclo menstrual y ovulatorio así como el interés por encontrar regularidades era, en realidad, muy alejado de lo que sucedía en los casos donde era posible que se presentaran, incluso, mujeres que nunca habían tenido períodos menstruales. Con ello, desafiaba toda lógica expuesta hasta el momento. Moreau afirmaba que, si bien se tendía a "reconocer la acción del cuerpo amarillo como causa determinante de los fenómenos catameniales", ésta no era la única que intervenía y convenía considerar "la acción de otras glándulas de secreción interna, especialmente tiroidea" (88). La autora presentaba un grado de acuerdo respecto de la asociación entre caracteres primarios -como la presencia de glándulas genitales- y los caracteres sexuales secundarios. Suscribía, también, a la idea de que a medida que se envejecía, el dimorfismo sexual tan elocuente en la juventud de todas las especies, mostraba signos de la asunción de caracteres 
masculinizantes en la mujer y femeninos en el varón (89). El caso de los eunucos o de las mujeres con ovariotomía era para ella elocuente de lo que producía la ausencia de marcadores sexuales definitorios y también avanzaba en el análisis del pseudohermafroditismo señalando la múltiple explicación del mismo. Sin embargo, luego de un repaso de la literatura sobre el tema, Moreau señalaba que la correlación entre las glándulas genitales y esos caracteres debía ser puesta en cuestión. Primero, señalaba otros factores endócrinos. Luego, afirmaba que, en relación a este tema, de todos modos, los hechos no eran "aún bastante numerosos ni claramente establecidos para fundar con bases sólidas una teoría" (93). Así, la inspección crítica, lógica y metodológica de la literatura existente sobre el tema le permitía arribar a ciertas afirmaciones. Aunque reconocía una asociación de la función endócrina del ovario con la nutrición (en particular, la obesidad), enfermedades dérmicas (en particular, manchas durante el embarazo), tomaba distancia respecto de las vinculaciones entre la función endócrina del ovario y alteraciones nerviosas (especialmente la neurastenia, psicostenia, manías, entre otras) u oculares y consideraba insuficiente la explicación de la actividad ovárica para explicar virilizaciones en pseudohermafroditas en la que consideraba que las glándulas suprarrenales tenían una importancia liminar, aunque planteaban el problema de casos de mujeres masculinizadas y varones superexcitados en sus apetitos masculinos. En su tesis sentenciaba "en verdad, los hechos son más complejos de lo que quisieran los que fundan y sostienen teorías" $(74)^{5}$. Su tesis propone repensar la función del ovario sin reducirla a la acción reproductiva. En ese sentido, discrepa con lo que era parte del sentido común de la ginecología de ese entonces "muller tota in uterus", pero también con la de a endocrinología "muller tota in ovaries' (Eraso, 2007).

Alicia Moreau integró la cultura científica de principios del siglo XX en el sentido que Oscar Terán otorgó a este concepto (2000: 11). En efecto, sus conocimientos científicos sirvieron para fundamentar sus intervenciones en sentido amplio. Su perspectiva imprimió una impronta ciertamente menos biologicista a sus consideraciones que la que los cánones sociales expresaban por entonces. Si para ella el cuerpo constituía un sustrato material inapelable y no quebró las visiones dicotómicas que atravesaron el pensamiento de la época tales como masculino-femenino; naturaleza-cultura; entre otras, referidos 
por Pablo Ben (2000), intuyó que no había tal reducto de las regularidades armónicas que se esperaban de los cuerpos sanos y que lo patológico tal cual lo expresaban las posiciones hegemónicas tenía menos que ver con las herencias que con el medio. De allí que, desde sus primeros pasos por el ámbito de formación académica, desplazó sus miradas hacia lo social y hacia la influencia que podía ejercerse sobre esos cuerpos que requerían, fundamentalmente, educación.

\section{La impronta del medio socio-educativo}

En el intenso año de 1906, Alicia, en compañía de su padre, Armand, ex comunero francés que constituyó una influencia notable en su pensamiento, asiste como ponente al Congreso Internacional del Libre Pensamiento, realizado en Buenos Aires el 20 de septiembre (Deleis et. al., 2001). Allí, expuso su estudio La Escuela y la Revolución, en el que retomaba parte de los argumentos esbozados en los artículos sobre psicología experimental. Comenzaba el artículo con el objetivo de "rechazar, destruir para siempre la creencia de la continuidad del pecado, la herencia del crimen, de la vergüenza, piedra angular del Cristianismo". Afirmaba que el crimen no se heredaba y consideraba al hijo libre de faltas de los antepasados (Moreau, 1909a: 5 y 6). Subrayaba la necesidad de una educación que abandonara el espíritu dogmático y los criterios de autoridad ajenos a la razón como los de la Iglesia cristiana, contra la que fustigaba constantemente evidenciando una profunda postura anticlerical que compartía no solo con su padre sino con varias compañeras de estudio y militancia feminista como María L. Berrondo o Julieta Lanteri, e hiciera valer el espíritu científico en la escuela. Seguía al filósofo italiano Andrés Angiulli (1837-1890) y su obra La Filosofía y la Escuela (1888), texto que admiraba6 .

Sorprenden en esa convicción, su defensa a ultranza de los “desvíos" revolucionarios, lejanos a las visiones más armónicas y pacifistas con las que comúnmente se asocia a esta figura: "Puede la revolución haber cometido sus errores, puede haberse desviado de su ruta alguna vez, pero cuando se pasa de las tinieblas a la luz ¿quién no queda cegado un instante? (...) ¿Qué son esos extravíos comparados con las magnas obras?" (Moreau, 1909a: 6). Sin embargo, y tal como señala Marina Becerra en relación al dirigente 
socialista Enrique Valle Iberlucea, en este momento consideraban que "no existían posibilidades reales de cambio social sin ruptura revolucionaria" (Becerra, 2010: 131).

Alicia mixturaba ideas que se correspondían con las lógicas de los mundos que transitaba. Su vocación docente la traslada a la Sociedad Luz donde, junto con Ángel Giménez, del Valle Iberlucea y otros referentes del socialismo argentino, dictaba cursos. Desde 1910, junto con su padre y Berta W. Gerchunoff, inaugura el Ateneo Popular, ámbito para la educación popular sobre temas como la prostitución, el alcoholismo y la prevención de enfermedades.

Publicada a partir de 1908, en un emprendimiento conjunto con del Valle Iberlucea, la Revista Socialista Internacional (RSI) marca la transición en la obra de Moreau. Muy colateralmente Alicia abordó en la revista temas que no fueran de educación o feminismo ${ }^{7}$. Los temas educativos son centrales en esa producción tanto en lo referido a la educación racionalista como a la educación popular y las actividades de extensión universitaria (Moreau, 1909e: 105-107). No serán los de feminismo sus textos liminares, sino algunos artículos sueltos a título informativo de un momento de creciente actividad y diseño de estrategias de visibilización que tuvieron a Alicia entre las más creativas de las organizadoras.

Decididamente, desarrolló allí las nociones sobre las que cimentaría buena parte de su ideario de los años posteriores y que le permitirían exponer las problemáticas de la educación. Asimismo, en este período, comenzó su actividad profesional tanto en el ámbito hospitalario como privado, multiplicidad de tareas apenas aliviada en un consultorio ubicado en el mismo inmueble donde ejercía el rol de secretaria de la RSI - posteriormente, devenida Humanidad Nueva (HN)-.

Luego de que en el primer número se reprodujera la disertación de Alicia en el Congreso del Libre pensamiento, siguieron otros títulos en la misma sección, cuyo contenido reforzaba las ideas de la pedagogía ya referidas y avanzaba en otras nuevas ${ }^{8}$. Consideraba que la educación racionalista no era sinónimo de laica y revelaba la importancia de la capacidad de preguntar que tenían los niños (Moreau, 1910b). Ella sostenía que era necesario desarrollarla e incentivarla en el conocimiento de la historia de la civilización de manera que los alumnos aprendieran de la historia de la humanidad, 
con los errores, pero también, apreciaran lo que había costado cada conquista (Moreau, 1909d: 11). También, señalaba que el cuerpo docente asumía una docilidad exasperante frente a las imposiciones oficiales (Moreau, 1910d: 302). Pero confiaba en la evolución y el progreso que proveía el espíritu científico. Éste, como vimos, operaría sobre lo innato y hereditario al punto tal de poder modificarlo. Ello debía animar la marcha hacia la consecución de los fines de una escuela racionalista que formara no solo al hombre -en sentido inclusivo de varones y mujeres pues postulaba la coeducación de los sexos- en conocimientos, sino en espíritu crítico y solidario, cuya moralidad no podía ser cuestionada -decía- por quienes pretendían que laicidad y racionalismo no implicaban valores morales (Moreau, 1909f).

$\mathrm{Su}$ primer texto sobre feminismo aparece cuando la revista muda su nombre a Humanidad Nueva. En ese artículo, Alicia analiza el modo en que la exclusión histórica de las mujeres de ciertos derechos -como la educación- no es un indicador de su incapacidad intelectual. Embiste contra quienes consideran que el desarrollo intelectual de las mujeres es una "monstruosidad biológica", causal de la crisis de maternidad (en alusión explícita a Leopoldo Lugones, un destacado escritor argentino, pero también, un ideólogo que viró su posición hacia una ideología de derecha) y los que prefieren como ideal "la cenicienta, descuidada o asesinada por la ley, pero robusta, sana y productiva, la mujer verdadera cargada de hijos". Antes que al intelectualismo, Alicia acusa a la religión cristiana de atentar contra la maternidad pues presenta como ideal "el renunciamiento a todo lo que hace la felicidad de la vida, idealizando la virginidad, poblando el mundo de conventos, presentando como horrendos pecados los afectos más puros" (Moreau, 1910a: 23, 27-29).

Moreau señala que, si bien el feminismo pudo adoptar formas llamativas (como la vestimenta masculina de muchas europeas), en realidad, "el feminismo no fue un detalle de indumentaria, sino una forma distinta de pensar; no se trató de oponer la mujer al hombre, sino de elevar, de esclarecer la primera, de no dejarla ajena al pensamiento moderno, de permitirle que conquistase en la sociedad una posición menos deprimida, de darle los medios de defenderse contra un régimen que no ha sido hecho para ella y en donde se encuentra herida y vejada cuando las circunstancias de la vida oblíganla 
a luchar a la par del hombre" (Moreau, 1910a: 29). Sin embargo, Alicia no cuestionaba la maternidad como destino de la mujer, sino la idea de que ella debía criar meramente. En efecto, para Moreau, la maternidad era un papel fundamental de las mujeres y su tarea iba acompañada de la educación de los hijos, no de su mera crianza o de la obligación de las funciones hogareñas (Moreau, 1910e). Desde esta perspectiva, atacaba la división sexual del trabajo en la sociedad capitalista y proponía, desde el punto de vista genérico, un método racional de organización de la domesticidad -abarcando, incluso, el plano arquitectónico- con el objetivo de reducir "el tiempo de las actividades rutinarias en un hábitat colectivo" (Vallejo, 2001). Con ese tiempo extra, las madres podrían desarrollarse en sus facetas personales y profesionales sin relegar las tareas de educación de los hijos, que también consideraba que debían asumir los varones.

Desafiando los imperativos excluyentes, no solo había ingresado a la carrera de medicina sino que había impulsado el Primer Congreso Femenino en la ciudad de Buenos Aires en 1910, formalmente organizado por la Asociación de (Mujeres) Universitarias Argentinas. Según concluía Alicia Moreau, "si en verdad la mayor parte de las declaraciones votadas son puramente... líricas, algo ha habido muy práctico: es el ejemplo dado, el camino abierto" (s / d b: 530) ${ }^{9}$. Señalaba, asimismo, la heterogénea composición de las integrantes, pero remarcaba el espíritu solidario que las había movido a unirse por un fin que anhelaba "el disfrute integral de la existencia" para las mujeres.

Para la década del10, Moreau se expresa a favor de la ciudadanía por etapas y aceptaba el voto municipal, más no el nacional ${ }^{10}$. Así, “expresaba dudas sobre la posibilidad de la ciudadanía femenina plena; todavía era presa de la convicción de que era más importante para las mujeres el asegurarse primero educación -tan coincidente con el molde del iluminismo liberador del socialismo-, ya que sólo así podrían acceder a la conquista de los derechos cívicos" (Barran$\cos , 2004)$.

\section{La pedagogía feminista-socialista}

En 1919, dejó de publicarse Humanidad Nueva y, ese mismo año, Alicia viajó a Estados Unidos a dos congresos. Uno, en calidad de 
médica y por el cual eleva un Informe al gobierno en el que hizo especial hincapié en lo referido a la salud femenina infantil, la educación sexual y sanitaria, la protección laboral y el papel del Estado (1922: 276-279).

Asimismo, asistió al Congreso Internacional de Obreras celebrado en Washington del que volverá compenetrada absolutamente con el ideal igualitarista, mudando su postura de sufragio por etapas del período anterior. Tras ese periplo, se distancia ideológicamente -aunque no políticamente- de figuras como Sara Justo que aún mantenía ciertas reservas con el carácter universal de los derechos políticos y, además, planteará algunas nociones menos diferenciales que las que podía esbozar su colega y cercana aliada en la lucha por los derechos femeninos, Elvira Rawson, más afecta a una mirada diferencial.

Vuelta de este viaje, impulsó y presidió la Unión Feminista Nacional que nucleó a distintas agrupaciones socialistas y feministas tales como el Centro Socialista Femenino y la Agrupación Socialista Femenina. Allí compartió escena con varias colaboradoras de RSI y HN como Julia García Games y Bertha de Gerchunoff. Asimismo, creará el Comité Femenino de Higiene Social cuyo objetivo es denunciar y eliminar distintas formas de "comercio de las mujeres" con fines de prostitución.

Se afiliará formalmente al Partido Socialista a comienzos de la década del 20. Poco después, cuando para los estereotipos de la época, Alicia parecía destinada a convertirse en una "feminista solterona", con sus 37 años se unió en matrimonio al fundador del Partido Socialista Argentino, Juan B. Justo, y fue madre. A partir de 1928, conservaría su labor profesional privada para sobrellevar las vicisitudes económicas de una viuda con tres hijos pequeños.

Durante la década del 20 y el 30 encontramos el momento más rico de Alicia en la producción sobre feminismo en general y sufragismo en particular. Participa en dos revistas: Nuestra Causa (1919-1921) y Vida Femenina (1933-1942). Este corpus documental ha merecido, recientemente, una consideración particular desde la historiografía de las mujeres (Montero Miranda, 2009; Masiello, 1997; Gallo, 2004).

En la publicación Nuestra Causa -espacio multipartidario amplio que reunía artículos sobre las temáticas de interés del feminismo de 
esos años particularmente vinculada a los derechos políticos y civiles así como a temáticas culturales (Jesús, 2010)-, Alicia muestra a lo largo de sus artículos las redes internacionales que las feministas iban conformando y, a la vez, avanza sobre el análisis de cómo es necesario modificar el medio para lograr la superación de la condición femenina, comenzando con experiencias locales -como el voto municipal- hasta llegar a otros niveles (Moreau, 1919; 1920a; 1920c; 1920d; 1920b). Nuestra Causa muestra el devenir de Moreau hacia las posturas igualitaristas de raigambre ilustrada. Asimismo, retoma las consideraciones fundamentales recogidas en el Congreso Internacional de Médicas cuyos núcleos, también fueron expuestos en la revista La Semana Médica a raíz del Informe presentado al Ministerio por Moreau -que ya analizamos-.

En esta década del 20, las posturas conservadoras compartían la politización de la maternidad a la que se apelaba desde el Estado y los movimientos feministas (Nari, 2000). Un discurso hegemónico sobre los roles sexuados y la división dicotómica de esferas comenzaba a resquebrajarse (Giordano, 2005). Si bien en los 30 no faltaron intentos de volver la situación atrás respecto de los logros para las mujeres como sujetos de derechos, lo cierto es que había una convicción creciente sobre la necesidad de una justicia reparadora de la exclusión de las mujeres de ciertos derechos como los políticos.

En esa línea, se posicionó el primer número de la revista Vida Femenina (1933-1942), un emprendimiento político cultural del Partido Socialista (Selene Román: 2010). María Luisa Berrondo fue la directora de este emprendimiento y Alicia Moreau de Justo fue una de sus más conspicuas colaboradoras. La propuesta de Vida Femenina estaba destinada a las "mujeres inteligentes" a quienes consideraba capaces de "intervenir en política y ocuparse de los problemas cotidianos como la salud familiar, la puericultura, la mejor alimentación para los niños y otros temas pertenecientes a la vida cotidiana femenina" (Rey, 2010). En esta revista, las producciones de Alicia son variadas e incluyen artículos heterogéneos que la posicionan como educadora, médica, feminista y socialista. En todos los casos, su mirada presente es crítica respecto de la coyuntura y los procesos socio-estructurales. Asimismo, confirma la seguridad en una evolución social de la civilización y, aún ante el flagelo de la guerra, considera que será posible una mejora a través de la educación que, 
sin duda, deberá llegar a las mujeres. Para entonces, además, Alicia ya había señalado la comunión de principios entre el socialismo y la defensa de la condición femenina (1933).

Por razones de espacio, no me extenderé en la obra escrita en estas revistas, sin embargo, retomaré el libro de Alicia Moreau La mujer en la democracia, publicado en 1945, pues resulta ser una síntesis de la labor de aquellos años. El título no es azaroso y devela que estuvo construido al calor de los tiempos. Según Marysa Navarro, este es "su último libro sobre feminismo" constituyendo a la vez "un alegato con el doble propósito de demostrar a sus compatriotas la necesidad de defender la democracia y sus principios, atacando toda forma de autoritarismo y también convencerlos de que para que la democracia fuera verdadera tenía que incluir a las mujeres" (1994: 191). Es una obra que podría considerarse como "bisagra" de la producción de Moreau pues amalgama la reflexión profunda y largamente realizada por esta pensadora así como las impresiones que el magmático entorno de los 40 imprimió a su pensamiento. Precisamente, el contexto de publicación de la obra es el segundo elemento que la hace atractiva para volver sobre ella. Publicada en agosto de 1945, permite avizorar la vivencia de un momento que, sin duda, ha marcado la historia de este lado del cono sur. El golpe de Estado de 1943 se había instalado como un nuevo quiebre político y el carácter ultraconservador sobre todo en cuestiones de género, conllevó una fuerte reacción por parte de los movimientos feministas locales. La mayoría de estos movimientos feministas estaban embanderados en la oposición "democrática" contra los gobiernos de facto surgidos en 1943 y visualizado como el signo de la presencia del nazifascismo en la Argentina (Bisso, 2002). Para esta oposición democrática (heterogénea y aglutinante de un amplio arco político-ideológico), el ascenso de Perón en ese gobierno no hacía más que confirmar aquellas sospechas. Especialmente, la izquierda alimentaba la idea del nazismo militar-peronista y ello la llevaba a autolegitimarse como vanguardia democrática. A principios de 1945, la promesa de un llamado a elecciones se vio acompañada por una incipiente apertura y movilizó políticamente a la sociedad. Sin embargo, acaece un hecho inusitado: por primera vez en la historia argentina, y suficientemente discordante en el contexto de un gobierno de facto, se propone desde una dependencia estatal -dentro de la Secretaría de 
Trabajo y Previsión- que el voto para la mujer fuera sancionado por decreto. Ello predispuso a buena parte de las sufragistas / feministas; mientras que otro grupo -no minoritario numéricamente- apoyó la medida. Moreau, claro, estaba entre las detractoras del "sufragio desde arriba". La movilización política encabezada en contra de Perón, promotor entre bambalinas de ese sufragio, terminó con lo que podríamos llamar un triunfo de las opositoras. Sin embargo, a la distancia, la victoria sería finalmente una amarga derrota. En efecto, si las sufragistas/feministas rechazaron el sufragio de un gobierno espurio y viciado en su origen por ser un golpe de Estado antidemocrático, poco pudieron esgrimir ante la propuesta cuando Perón ha sido electo en una de las elecciones más limpias en años.

A escasos días de aquellos convulsionados acontecimientos, Moreau publica -en 1945- La mujer en la democracia. La obra presenta un recorrido que se vertebra en torno a un antagonismo, esencializado y moralizado: "Democracia" / "Autoritarismo". Para Moreau, democracia es un estilo de vida donde la clave la da el compromiso de cada ciudadano para con el sistema de gobierno: “... la democracia y la autocracia no son simplemente formas de gobierno, sino que traducen modalidades anímicas del ser humano, que son la expresión política de tipos de hombres que conviven..." (1945: 16). Alicia Moreau retoma aquí sus argumentaciones sobre el papel de la educación como punta de lanza para la construcción del estilo de vida democrático que logrará superar el "sintoma de inmadurez democrática" (1945: 112). La "alfabetización política” debía llevarla adelante la elite más educada y los educandos serían luego agentes multiplicadores. Esta preocupación era concurrente con la del Partido Socialista (PS) (Altamirano, 2002: 229).

En la obra, el recorrido democrático presenta una tensión entre la voluntad individual y los procesos evolutivos. Por un lado, para Moreau, la evolución democrática estaba signada por etapas jerárquicas siendo deseable alcanzar las superiores. La superioridad de la democracia del presente respecto de la del pasado encierra más que un desdén por aquello, un optimismo por lo que vendrá (1945: 15). Por otro lado, la democracia como campo de realización de las voluntades individuales genera el bien común. El sujeto político de la democracia moderna, idealizado en el ciudadano, no se define solo por el acto de votar sino también por su preocupación por delimitar 
los intereses de la vida compartida con los conciudadanos. La idea de pacto restituye a los individuos el carácter volitivo y activo y, así, la libertad individual ilustrada garantiza la pervivencia del sistema democrático e involucra la formación ética y moral claves de los procesos de socialización y de sus mediadores.

Cómo acercar el feminismo a potenciales lectores no solo desprevenidos sino, además, negativos hacia lo que en el sentido común figura como un intento de subvertir las jerarquías sociales tal como se conocieron desde siempre. Cómo, en el contexto imperante, quebrar la misoginia de un gobierno que había reducido las expresiones democráticas. A lo largo de dos capítulos, Moreau se explaya sólidamente sobre el feminismo con la contundencia de sus cuarenta años de militancia. Define que "el movimiento feminista es un movimiento social organizado con el propósito explícito de reformar la legislación, de abrir carreras, de mejorar las condiciones de trabajo y hacer desaparecer los prejuicios y las prácticas que impiden a la mujer desenvolver su vida con libertad y sin más limitaciones que las que nacen de sus naturales condiciones individuales". La autora continuaba con una definición por negación que, a la vez, evidenciaba la maliciosa lectura que algunos realizaban de este movimiento: "No es, como equivocadamente suponen algunos, la guerra de la mujer contra el hombre, el desplazamiento de éste o la igualación contraria a la naturaleza misma" (1945: 34). De este modo, articula el feminismo con el movimiento general de los pueblos hacia su perfeccionamiento civilizatorio: la democracia. A partir de esta definición, la lucha feminista es una empresa en la que es posible encontrar a varones y mujeres por el derecho de participación de la mujer.

La dirigente explota la riqueza potencial del concepto de igualdad: "igual en derechos, en oportunidades, en tarea, en responsabilidad. Cuanto antes se llegue a esta igualdad -que es social, no biológica ni psíquica- antes se alcanzará un estado superior de entendimiento y armonía entre los grupos humanos" (1945: 273-274). De haber tenido derechos políticos tempranamente, las mujeres "habría[n] cometido errores, los mismos que ha cometido el hombre, pero juntos los hubieran rectificado y juntos hubiesen aprendido" (1945: 27). Existen tantos varones como mujeres poco capacitados para el ejercicio de las prácticas democráticas. Ello debido a que las diferencias no son en razón de lo biológico sino de la socialización. 
Su diagnóstico de la situación femenina reconoce las limitaciones que la socialización imprime en las prácticas democráticas diferenciando varones y mujeres pero también señala lo que pueden ser las puntas del iceberg sobre las cuales edificar una democracia plena: la inserción en el mercado de trabajo y la redefinición de la maternidad a través de la educación.

Desdobla la gestación, función biológica, del cuidado y educación: "lo que puede cambiar el sentido de la civilización no es pues esa fuerza que asegura la persistencia de la especie, sino la conciencia de la responsabilidad ante la vida" (1945: 266). Retoma sus ideas de principios de siglo cuando postulaba que la mujer no era mera reproductora y niega que la condición biológica de gestar conlleve el "instinto materno" de protección y cuidado. Varones y mujeres poseen esa conciencia de cuidado pues es socialmente adquirida y verdadera condición de la vida de la especie humana (267-270). Apoya sus apreciaciones en una lúcida percepción de los cambios demográficos y, sin alarma ante la denatalidad -la cual tenía convulsionados a los contemporáneos (Ramacciotti, 2003), anuncia un nuevo tipo de familia basado en la responsabilidad procreadora que a su vez augura interrelaciones que permitirán mayor contacto afectivo entre padres, madres e hijos.

Sin embargo, tensando esas ideas, las mujeres entrarán en la democracia no a través de la ruptura de la identificación maternidadcuidado-educación sino por su profundización (Lionetti, 2003): “Lejos, pues, de ser la maternidad plenamente cumplida un obstáculo para la función política, diremos que casi es su mayor razón de ser, y que tanto más alta sea la conciencia de su responsabilidad materna, más querrá la mujer poseer los medios de acción colectiva que le permitan sobrellevarla mejor" (1945: 196). Desde allí se construirá la nueva ciudadanía femenina revalorizando el rol cuidadora-educadora (1945: 32). Moreau no niega la tarea de cuidadora sino el sentido de la educación y, a la vez, también reconoce un papel al varón en esa tarea. Como señala Nari, Moreau retoma los sintagmas de las feministas que "intentaron reformular la maternidad. No cuestionaron que constituyera una 'misión natural' para las mujeres; pero fundamentalmente la consideraron una 'función social' y, para algunas, incluso, una 'posición política'...” (2000: 205). 
Además de la incorporación laboral, las mujeres deberían, aún sin acceder al voto, incluirse en los partidos políticos, canales de expresión propios de los sistemas democráticos (1945: 234). La formación y la legislación -en ese orden- confluirían en el reconocimiento de la capacidad ciudadana femenina. A diferencia de lo que postulaba en la década del 10, consideraba entonces que "el sólo hecho de votar significa para quienes no lo han hecho nunca un cambio irreversible, un paso hacia la liberación de la conciencia, la adquisición de una responsabilidad nueva" (1945: 126). Al perfeccionarse la vida democrática no existirá una práctica diferencial entre mujeres y varones quienes votarán y serán representantes de otras mujeres y varones indistintamente y gobernarán para lograr su bien común.

Teniendo en cuenta la coyuntura local, en la que dentro del gobierno de facto se distingue la figura ascendente de Perón, la autora se pregunta cómo evitar que en Argentina vuelvan a surgir modelos como el de los golpes militares filo nazis. La dirigente socialista rescataba en Argentina una estructura democrática que la diferenciaba de las traumáticas experiencias europeas (1945: 16) y que sería la que presionó contra los grupos autoritarios en el poder para que desistieran del mismo. Para ella, en ese momento y en la evolución de los sistemas políticos mundiales, Argentina estaba en proceso de ascender a una etapa más democrática. Para concurrir a la formación de la vida democrática es imperioso que se eduque a los habitantes, varones y mujeres, y a la mujer en particular pues "nadie se interesa por el ejercicio de un derecho que no tiene" (1945: 30-31). En plena algarabía ante los futuros comicios, ella alerta sobre el peligro de una democracia formal montada sobre la mera gestualidad electoral. Avanza sobre un doble problema. Uno, un problema unido al sistema político: cómo contraponerse a la conceptualización de ciudadanía y democracia articulada en discursos como el de Perón que no consideran la construcción de las mismas apoyándose en el individuo libre y autónomo que para Moreau sigue siendo el referente de ciudadanía. El otro problema está referido a la cultura política: cómo tener una práctica democrática cotidiana si la socialización de los ciudadanos y ciudadanas está basada en formas que no se corresponden. La falacia democrática se evidenciaba en la exclusión de la mujer como forma evidente y solapaba un problema más amplio, el de la educación ciudadana. 


\section{Antiperonismo y tensiones en el ideario democrático}

El triunfo de Juan Domingo Perón en las elecciones de 1946 fue el comienzo de un cambio en la organización política. La acción expansiva del peronismo abarcó especialmente a las mujeres tanto en el intento de organizar su participación a través de las vías partidarias como en la preocupación por dictar un estatuto normativo sobre los alcances de sus derechos políticos. Sobre el primer aspecto, rápidamente alcanzan visibilidad los centros cívicos, que luego se unificarían en el Partido Peronista Femenino (1949) (Barry, 2009). A su regreso de Europa, en 1947, Evita se erigió en adalid de los derechos políticos de la mujer y potenció la necesidad de sancionar una normativa legal para enmarcar esos derechos (Valobra, 2010). La ley 13010 de derechos políticos de la mujer abrió un juego político en el que las mujeres se convertían en una apetecible "clientela política".

Tras la derrota electoral de 1946, el Partido Socialista encarnó una acción de sistemática oposición (Camarero y Herrera, 2005; García Sebastiani, 2002), aunque por primera vez, sin representantes parlamentarios. La falta de bancadas parlamentarias y las dificultades del accionar público por la persecución sufrida por el partido le quitarán posibilidades de intervención política. Según Marcela García Sebastiani (2002), a raíz de su nueva situación postelectoral el socialismo debió apelar a una estrategia de oposición basada en actos públicos y la edición y la opinión crítica al gobierno desde La Vanguardia como una manera de mantenerse en el juego político visible en la esfera pública. El socialismo apostó a reforzar los dispositivos educativos y propagandísticos a fin de "ilustrar" al pueblo para que finalmente comprendiera que su partido de clase no era el peronismo sino el socialismo.

Alicia se inscribió en esa línea y aceptó, fiel a sus principios, las consecuencias de las elecciones de 1946 aunque no hubieran resultado a favor de su partido como anhelaba. Su postura antiperonista y la evaluación del momento político como condensación autoritaria iban contra todo pronóstico optimista que apenas un año atrás había formulado respecto del futuro político nacional. Pero, después de todo, ella misma había sentenciado que un pueblo poco educado en las prácticas y teorías democráticas pudiera elegir a quienes mejor lo representarían. Si bien había implícita una desconsideración a la 
elección realizada por el pueblo y una autoimagen de vanguardia política propia del pensamiento de la dirigente, Moreau trabajó para elevar la cultura ciudadana, especialmente la femenina que se veía amenazada por la manipulación que se pretendía hacer del voto femenino y el avance de Evita, esposa del primer magistrado, vista por el socialismo como una cortesana, revanchista y antojadiza. Para Moreau esta ley adolecía de tres males: era sancionada en un contexto profundamente antidemocrático, el peronismo; pecaba de una intención claramente clientelística y atribuía el éxito a una recién llegada sin reconocer la lucha sufragista anterior (Henault, 1983: 107). Sin embargo, ni individualmente ni a nivel partidario rechazó la ley de derechos políticos femeninos cuando fue sancionada en septiembre de 1947.

Desde el Partido, del que Alicia formaba parte del Comité Ejecutivo desde la década del 30, se lanzó una campaña de educación ciudadana. En la "Conferencia Nacional de Mujeres Socialistas" de 1947, Alicia invitó a las mujeres a "crear a la sufragante" con conciencia (1947: 1). Las militantes comenzaron así prácticas didácticas realizadas a través de giras, charlas y debates por medio de los que intentaba esclarecer a las argentinas para que el voto no sirviera "de pedestal a los 'salvadores de la patria' con alma fascista" (La Vanguardia Femenina, 1946: 9). También publicitó las iniciativas socialistas y visibilizó a sus propulsores, construyó sus propias tradiciones y liderazgos y destacó también a personalidades extrapartidarias ${ }^{11}$. Las socialistas se mostraron activas en educar a las futuras ciudadanas con campañas de distinto alcance que incluían los envíos por correo de artículos, charlas y conferencias.

Sin embargo, la persecución del gobierno obligó a los y las militantes socialistas a una acción semiclandestina, cuando no clandestina. Los escritos de Moreau menguaron en este lapso. Sus obras de este período apuntan, fundamentalmente, a la consolidación del partido y a delinear propuestas orgánicas de funcionamiento. Tal el caso de su obra El Socialismo según la definición de Juan B. Justo de 1946. Seis años más tarde, al tiempo que el Partido se sumía más y más en una crisis, la veterana dirigente creyó oportuno apuntalar las nociones de Partido. Antes de las elecciones de 1951, Moreau dictó una conferencia en la que se preguntaba ¿Qué es un partido político? Allí, discurría en la diferenciación entre un partido democrático y 
uno autocrático, no siendo este último, en realidad, un verdadero partido sino un instrumento personal de un tirano. A lo largo del texto se hacía alusión implícita al peronismo. Así, se señalaba que "ningún político, por grande que sea su influencia" podía hacer abstracción de una tradición democrática cimentada a lo largo de la historia. Moreau insistía en rescatar para nuestro país un ideal democrático que sería, finalmente, salvaguarda del futuro y sobre el que cimentaba la dirigente el optimismo en las instituciones de libertad y bastión de la misma: el sufragio universal (1952: 15).

Ello, con todo, no la exceptuaba de lecturas elitistas: "[los partidos] agrupan los hombres y mujeres más activos, los que comprenden con más claridad la inquietud que los grandes problemas colectivos despiertan" (1952: 10). Los políticos como un baluarte moral de la nación aparecen repetidamente en esta obra de Moreau. Su concepción del partido implicaba una organización de cierto grupo selecto en el cual la población delega ciertos aspectos del manejo de la cosa pública hizo que la lectura de sí de los socialistas tergiversara su propio objetivo social y político. Como señala García Sebastiani (2002), ello impidió al partido revisar sus propias bases programáticas.

En los escritos de este período, Moreau afila la idea de que el socialista es un partido de clase obrera, aunque "su composición humana puede no serlo" (1952: 34). Proponía que el Partido conservaba la representación obrera aún cuando los obreros no lo hubieran votado por falta de comprensión sobre cuáles eran los medios para alcanzar sus ideales "como clase" (1952: 12-13). El tono propedéutico, elitista y desconsiderado de la racionalidad de los electores le quita el distanciamiento y plasticidad a su conceptualización de la política que se había apreciado en otros escritos. Devela el intento desesperado por dar vida a un partido que languidecía irremediablemente no solo por la supuesta falsa conciencia de los votantes sino, también, por las estrategias de represión que recayeron sobre el mismo ${ }^{12}$.

En ese marco, Alicia se preocupó por la apertura a las nuevas generaciones a quienes era necesario alejar de la influencia peronista que tanto influjo tenía. Cuando Alicia Moreau publica su obra La juventud argentina y el Partido Socialista, en 1953, vuelve a los mismos tópicos de esas dos obras mencionadas en el párrafo anterior. La propuesta de una vida deportiva y política se aunaban en la propuesta que intentaba captar a los jóvenes (de sexo masculino) y orientarlos 
por una senda que los alejara de la fascinación del peronismo y sus propuestas deportivas grandilocuentes. No obstante, contrariamente a lo que se ha postulado en relación con el distanciamiento que los viejos dirigentes presentaban respecto de la juventud (Tortti, 2009), Alicia abre un camino en relación con las nuevas generaciones que la colocan nuevamente a la vanguardia de las estrategias políticas del socialismo. Más allá de que los resultados fueron dispares en relación con esa intervención, cabe señalar que ella es también tributaria de la posición marginal que Alicia mantenía en el partido, a pesar de encontrarse en órganos de decisión del PSA. Durante este lapso, sus apuestas feministas -que habían caracterizado su labor durante los períodos anteriores- quedaron subsumidas a los denodados intentos de brindarle soportes de subsistencia al partido.

Luego del golpe de 1955 que desalojó del gobierno a Juan Domingo Perón, la Dra. Moreau de Justo fue designada por el Comité como integrante de la Junta Consultiva Nacional (JCN), un organismo creado por el gobierno de facto conformado por representantes de distintos partidos (excepto el peronista y el comunista) que debían establecer su opinión sobre ciertos puntos sobre los que el ejecutivo se expediría luego. En ese contexto, el PSA constituye para María Spinelli un partido revanchista en tanto alienta el castigo de las clases ilustradas sobre la plebe peronista (133). Moreau esgrime que en ese momento y teniendo en cuenta que el gobierno peronista representaba una tiranía nazi fascista, "lo único que se puede pensar: desmantelar la máquina construida por la tiranía, educar al pueblo en la sana doctrina de las convivencias, elevar su standard de vida y sustraerlo de la posibilidad de que cualquier especulador, por inteligente que sea, lo aproveche, lo explote y lo convierta en instrumento ciego de sus ambiciones" (El Litoral, 1956: 4). Ello genera una tensión respecto de sus posiciones sobre los gobiernos militares que antes había censurado. Introducida la idea de que el peronismo era una tiranía, salvaba -al igual que lo hacía el partido-, pero no sin inconvenientes, al gobierno militar de la Revolución Libertadora. Sin embargo, en algunos momentos, Alicia se mostrará incómoda con este rol en la $\mathrm{JCN}^{13}$. A su vez, tendrá posiciones diferenciadas de los otros consejeros socialistas en la JCN, especialmente cuando se debate sobre la intervención política de las mujeres que se reedita en torno a los 
problemas de su empadronamiento y a la reforma de la ley electoral (Junta Consultiva Nacional, 1956: 154; La Prensa, 1956: 1).

Las internas frente a la proscripción del peronismo y a la represión del gobierno de las manifestaciones de ese cariz, resquebraron al socialismo (Tortti, 2009). Además de dirigir el periódico La Vanguardia, del que es retirado Américo Ghioldi -quien junto con Repetto conformaban una línea opuesta a Moreau de Justo-, Alicia impulsa la revista Ciudadana, una revista mensual editada por la Unión de Mujeres Socialistas. Esta revista fue un intento por avanzar en la formación de las mujeres en el compromiso de la ciudadanía y, de alguna manera, de borrar la impronta nociva que había significado el peronismo sobre ellas. Apareció en marzo de 1956 y dejó de salir en diciembre de 1957 (Faure, 2010). Allí, junto con viejas compañeras de militancia como María Luisa Berrondo, Alicia comparte espacios con las nuevas generaciones que encuentran en la revista un ámbito para difundir ideas sobre diversas temáticas. Desde allí, además de apelar a la formación de las mujeres en las lides cívicas, se impugnaron algunas acciones del gobierno. Una de ellas fue la crítica por la suspensión de la ley de divorcio que desde Ciudadana se consideraba un atropello a la laicidad del Estado y que implicaba, también, un retroceso desde la mirada de género. Alicia escribió por entonces una contribución en la que sentenciaba: “¿Urgía que el gobierno provisional tomara tal medida que perturbaba a millares de hogares? Si hemos de llegar, dentro de poco tiempo, a un parlamento surgido del pueblo - ¿no se podía esperar que los distintos partidos políticos-incluyendo en sus respectivas plataformas electorales, la ley de divorcio o su negación, consultaran libremente la opinión pública?"14. Según la autora, la medida "por su rigidez" tenía "visos de crueldad" (1956a: 5).

La situación se presentó más compleja de resolver ante algunos hechos. Por ejemplo, en mayo de 1956 criticaron con contundencia los apremios policiales perpetrados contra un supuesto militante peronista (Ciudadana, 1956a). Sin embargo, cuando se produjeron los fusilamientos por el levantamiento pro peronista de junio de 1956 el editorial de julio apeló a la solidaridad de las familias antes las fuerzas de la regresión agazapadas (Ciudadana, 1956 b). Alicia no firmó una nota sino hasta el número siguiente, de agosto, donde sostuvo que el deber de la hora era "Impedir por todos los medios que el 
totalitarismo pueda volver", pero advirtió que no siempre era "fácil conocer los medios de cumplimiento de ese deber" por lo que era necesario comprender que el retorno al pasado "no puede ser impedido sólo por la presión armada, por la vigilancia permanente, por la angustia siempre lista a la defensa" (1956b: 22-23). Era, en verdad, muy difícil para Alicia congeniar sus ideales con el gobierno provisional que supuestamente venía a realizarlos. Seguramente, como lo revelan sus escritos, lo intuía, pero su abominación del peronismo la hacían persistir en que el camino estaba trazado y su intervención lo prohijaba, con una mirada severa, pero que no condenaba.

No obstante, como ya han detallado algunas investigaciones (Tortti, 2009), las críticas de las líneas internas que cargaban contra las antiguas generaciones en la dirección del partido. Alicia será blanco de las diatribas, incluso, cuando de algún modo amparó a esas nuevas generaciones. Luego, sobrevendrá el cisma del socialismo y es aquí donde concluimos este primer tramo del recorrido de la figura de Alicia Moreau.

\section{Consideraciones finales}

A lo largo del trabajo intentamos rescatar la individualidad, el talento y la originalidad de la pluma de Moreau de Justo. Hemos señalado la variedad y heterogeneidad de sus escritos, así como hemos marcado, también, un desplazamiento temático a lo largo de más de cincuenta años de producción revisitada.

A lo largo del período, ella va construyendo su visión de la mujer como individuo, como sujeto, como ciudadana, como militante. Al plantear el ingreso de las mujeres en la vida democrática, les impone una tarea más: la de educar al soberano de la vieja fórmula sarmientina en versión doméstica maternal. Sin embargo, Moreau no advierte que recarga a la mujer con nuevas actividades, tal vez porque ella misma fue un ideal de este tipo femenino que propiciaba (Lavrin, 1997: 179). Por otro lado, las estructuras patriarcales del ámbito familiar no son directamente atacadas sino a través de la lenta acomodación de las prácticas que, al permitir a las mujeres decidir principalmente a través del sufragio, van minando las resistencias a dicha decisión en otros planos. 
Hemos señalado, también, la lucidez intelectual y la capacidad para adelantarse y proyectar ciertas problemáticas que más adelante cobraron importancia. En este sentido, puso en cuestión la concepción de la igualdad misma y perfiló la idea de diferencia en muchos aspectos aunque su eje fuera un feminismo de la igualdad. Por otro lado, desentrañó la interrelación entre una construcción identitaria de la mujer y una adscripción subjetiva propiciada por los procesos de socialización. Entendiendo que era allí donde debían avanzar las luchas feministas, colocó en el centro de la cuestión pública una temática considerada exclusiva del mundo privado: el cuidado y educación de los hijos. No avanzó en la subversión de este orden sino que presupuso una transformación por un cierto efecto lineal "de contagio". Así como las mujeres aportarían su don esencial a la esfera política, el cuidado de la vida, ésta lograría una completud identitaria en las mujeres al elevarlas al rol de ciudadanas.

Los avatares políticos, la alternancia cívico militar, marcarán contradicciones, posicionamientos equívocos, silencios prolongados, colocarán a esta médica, educadora, intelectual y política argentina en situaciones contradictorias que tensarán su propio posicionamiento frente a las expectativas de la democracia, pero aún cuando jugaran en contra de sí misma, ella aceptará el lugar que le toque ocupar si ello tiene que ver con aceptar el florecimiento de los ideales democráticos como estilo de vida.

Alicia Moreau de Justo es una figura que abrió caminos e instaló problemáticas a través de su práctica comprometida con la realidad que a lo largo de su vida le tocó vivir. Este estudio ha querido señalar algunos aspectos de su actuación e idearios que apenas si comienzan a vislumbrar la complejidad de sus posicionamientos.

\section{Notas}

1 Agradezco la inapreciable colaboración de Gisela Manzoni y Verónica Norando en la asistencia a la investigación para este trabajo y a Nadia Ledesma Prietto por su atenta lectura. Asimismo, el inestimable aporte del Archivo Palabras e Imágenes de Mujeres del Instituto Interdisciplinario de Estudios de Género de la Facultad de Filosofía y Letras de la UBA (APIM) -especialmente a Ana Lía Rey al facilitarme el acceso a la revista Ciudadanas, un corpus documental inapreciable. 
2 Para abordar el origen del término intelectual y su desarrollo ver Altamirano, 2006.

3 En otro estudio hemos abordado la asociación que se hizo entre Alicia y la Eugenesia, la cual tampoco encuentra asidero, especialmente para épocas posteriores. Ledesma Prietto y Valobra, en prensa.

4 Alicia Moreau. La función endócrina del ovario, Tesis de grado, 1913, 57. El problema de los métodos era central para Alicia pues definían el acercamiento científico en el más estricto sentido positivista. Ya lo había planteado también en "Estudio experimental de la acción de la digitalina".

5 Algunos trabajos en el marco de las Cátedras diversificaron su producción y analizó las ventajas y desventajas del uso de ciertas sustancias o métodos de indagación, pero siempre con un profundo sentido crítico de los métodos. Ver Nerio Rojas y Alicia Moreau, 1909.

6 En 1907, había sentenciado la importancia de la educación, aunque no descuidaba los aspectos sensibles mixturados con los científicos: "la educación para ser fructífera debe comenzar con la vida, y esto se comprende, pues las primeras enseñanzas son las más duraderas, se imprimen en la época de mayor plasticidad cerebral, si así puede decirse, y todo lo que vendrá más tarde se asentará sobre esta base (...) De ahí la importancia de la educación familiar y primaria" (Moreau, 1907: 21-22).

7 Entonces, tocó cuestiones de higienismo como las condiciones del espacio laboral para garantizar la salud (Moreau, año 2, 15 de abril de 1910).

8 Sus aportes fueron, Moreau, RSI, año I, n 3, 15 de febrero de 1909; RSI, año I, nº 4, 15-18 de marzo de 1909; Año I, tomo II, nº 1, 15 de junio de 1909.

9 A partir de convertirse en Humanidad Nueva, la revista deja de precisar la fecha de publicación por lo cual no se consigna.

10 El Argentino, Chascomús, 5 de julio de 1914, 3.

11 La Vanguardia (LV), 5 de marzo de 1946, 5. LV, 20. LV, 27 de agosto de 1946, 6.

12 En 1951, ante las primeras elecciones en las que participan las mujeres a nivel nacional, Moreau de Justo no puede asistir como votante aunque es candidata a diputada por la circunscripción 16 de la Capital Federal. Hay sobre ella orden de prisión y permanece en la clandestinidad junto con otros opositores políticos. Obtuvo, con todo, 1074 votos.

13 Así, respecto de un comentario del representante del partido Unión Federal Demócrata Cristiana, Luis María Bullrich, quien señala que en ese organismo es la única mujer designada por el gobierno en la Junta. Moreau intenta, sin éxito, refutar esa supuesta designación a modo de deslindarse de una representación de corte verticalista. Señala "Yo no he sido designada por el gobierno (...) fui designada por el partido". Pero prontamente se llama a silencio cuando Bullrich afirma que fue designada "por sugestión del partido, pero con la firma del gobierno" (Junta Consultiva Nacional, 1956: 100).

14 Especialmente los números 1 a 3 de Ciudadana donde además de editoriales y notas de las integrantes del Comité de Redacción también escribió Silvina Bullrich y se publicaron reportajes de Elena Gil a mujeres que apoyaban la ley de divorcio. También, en diciembre de 1957 promovieron un número sobre ese punto de cara a las futuras elecciones. 


\section{Bibliografía}

Altamirano, Carlos. "Ideologías políticas y debate cívico". En Juan Carlos Torre (dir.). Los años peronistas (1943-1955). España: Sudamericana, 2002.

-----. Intelectuales. Notas de investigación. Buenos Aires: Editorial Norma, 2006.

Ascolani, Adrián. “Ciencia y moral en la escuela primaria: instrumentos psico-pedagógicos para el control de las emociones en argentina (primera mitad del siglo XX)". En Simposio Internacional Proceso Civilizador, Universidad de Buenos Aires, 2008.

Barrancos, Dora. “Ciudadanía femenina en la Argentina. Debates e iniciativas en las primeras décadas del Siglo XX". En Hugo Biagini y Arturo Andrés Roig. El pensamiento alternativo en la Argentina del siglo XX: identidad, utopía, integración (1900-1930). Buenos Aires: Biblos, 2004.

Barry, Carolina. Evita Capitana, el Partido Peronista Femenino 1949-1955. EDUNTREF, Caseros, 2009.

Becerra, Marina. Marxismo y feminismo en el primer socialismo argentino. Enrique del Valle Iberlucea. Rosario: Prohistoria ediciones, 2009.

Ben, Pablo. “Cuerpos femeninos y cuerpos abyectos. La construcción anatómica de la feminidad en la medicina argentina". En Fernanda Gil Lozano, Valeria Pitay Gabriela Ini,(comps.). Historia de las Mujeres en la Argentina. Buenos Aires: Taurus, 2000.

Biagini, Hugo. "Introducción”. En Hugo Biagini y Arturo Roig (dirs.). El pensamiento alternativo en la Argentina del siglo XX: identidad, utopia, integración (1900-1930). Buenos Aires: Biblos, 2004. http://www.cecies.org/imagenes/edicion_146. pdf

Bisso, Andrés. “De Acción Argentina a la Unión Democrática: el civismo antifascista como prédica política y estrategia partidaria del Socialismo Argentino (1940-1946)". En Prismas. Revista de historia intelectual, UN de Quilmes, n 6, 2002, 257-264.

Bourdieu, Pierre. "La ilusión biográfica". En Razones prácticas. Sobre la teoría de la acción. Barcelona: Ed. Anagrama, 1997.

------. Campo de poder, campo intelectual. Buenos Aires: Montressor, 2002.

Camarero, Hernán y Carlos Miguel Herrera. “El Partido Socialista en Argentina: nudos históricos y perspectivas historiográficas". En El Partido Socialista en Argentina. Sociedad, politica e ideas a través de un siglo. Buenos Aires: Prometeo, 2005.

Cichero, Marta. Alicia Moreau de Justo. La historia privada y pública de una legendaria y auténtica militante. Buenos Aires: Planeta, 1994.

Deleis, Mónica; Ricardo de Tittoy Diego Arguindeguy. Mujeres de la política argentina. Buenos Aires: Aguilar, 2001.

El Argentino. Chascomús, 5 de julio de 1914, 3.

El Litoral. "La Dra. Moreau de Justo habla de política. Claridad en los procedimientos y honradez en la acción", Miércoles 2 de Mayo de 1956, 4.

Eraso, Yolanda. "Biotypology, Endocrinology and Sterilization: The Practice of Eugenics in the Treatment of Argentinian Women during the 1930s'". En Bulletin of the History of Medicine, 81, 4, 2007, 793-822. 
Faure, Patricia. Ciudadana (1956-1957), Revista de la Unión de Mujeres Socialistas, en X Jornadas de Historia de las Mujeres V Congreso Iberoamericano de Género, Luján, 16, 17 y 18 de septiembre de 2010.

Gallo, Edith. Nuestra Causa, Revista Mensual Feminista, 1919-1921, Estudio e Índice General, IIHCS, Bs. As., 2004.

García Sebastiani, Marcela: "El Partido Socialista en la Argentina peronista: oposición y crisis de representación política (1946-1951)". En EIAL, volumen 13, número 2, julio-diciembre 2002.

Giordano, Verónica. “Los derechos civiles de las mujeres y la reforma del Código Civil de 1936: el acontecimiento, la coyuntura, la estructura". En Terceras Jornadas de Jóvenes Investigadores del Instituto de Investigaciones Gino Germani, Universidad de Buenos Aires, Facultad de Ciencias Sociales, 29 y 30 de septiembre de 2005. www.iigg.fsoc.uba.ar.

Girón, Álvaro. “Darwinismo, darwinismo social e izquierda política (1859-1914): reflexiones de carácter general". En Marisa Miranda y Gustavo Vallejo (comp), Darwinismo social y eugenesia en el mundo latino. Argentina: Siglo XXI, 2005.

Gómez Rodríguez, Amparo. "Ciencia y valores en los estudios del cerebro". En Arbor, CLXXXI n ${ }^{\circ}$ 716, 2005.

Henault, Mirta. Alicia Moreau de Justo. Buenos Aires: CEAL, 1983.

Huertas García-Alejo, Rafael. El delincuente y su patología. Medicina, crimen y sociedad en el positivismo argentino. Madrid: CSIC, 1991.

Iglesias Aparicio, Pilar. "La mujer según la ginecología del siglo XIX". http: / / www. ciudaddemujeres.com/articulos/La-mujer-segun-la-ginecologia-del

Jesús, Lorena. “Feminismo y política. La experiencia de la revista Nuestra Causa. Buenos Aires, 1919-1921". En X Jornadas de Historia de las Mujeres V Congreso Iberoamericano de Género. Luján, 2010.

Junta Consultiva Nacional, noviembre de 1956, Tomo I.

La Prensa. "El estudio de los padrones prosiguió ayer", 11 de abril de 1956, primera plana.

La Vanguardia Femenina (LVF), "Exitosa jira de L. Barrancos y H.M. Ricci”, 19 de noviembre de 1946.

La Vanguardia. "28 de junio de 1946 Cincuentenario del PS", 20.

-----. “El Dr. Palacios Historió los esfuerzos de nuestro partido, Acción legislativa Labor de la Dra. A. Moreau de Justo", 27 de agosto de 1946, 6.

------. "El senador socialista E. del Valle Iberlucea. Una vida ejemplar, consagrada al bien del pueblo", por Agustín Arrieta,

------. "La presencia de J. Marpons destacan en la capital venezolana", 5 de marzo de 1946, 5.

Lavrin, Asunción. “Alicia Moreau de Justo: feminismo y política, 1911-1945”. En Bárbara Pottast y Susana Menéndez. Cuadernos de historia de América Latina. Málaga, AHILA / Algazara, 1997.

Ledesma Prietto, Nadia y Adriana Valobra. "Lecturas eugénicas sobre la vida privada y social de las mujeres en la perspectiva de intelectuales del campo médico y político argentino, 1930-1945". En Gustavo Vallejo y Marisa Miranda. Políticas públicas, vida privada y control social y las redes eugénicas del mundo latino (en prensa). 
Masiello, Francine. Entre civilización y barbarie. Mujeres, nación y cultura literaria en la Argentina moderna. Rosario: Beatriz Viterbo, 1997.

Montero Miranda, Claudia. "Revistas feministas en Chile y Argentina: escrituras de y para mujeres en los años de entreguerras". En Nuevo Mundo Mundos Nuevos, Debates, 2009. URL: http:/ / nuevomundo.revues.org/57693

Moreau, Alicia. “¿Es necesaria una psicología que complemente la psicología experimental?". En El libro, Tomo I, 1906.

-----.. "La ciencia y la educación". En Universidad popular III, 1907.

------. “La vida y los fenómenos psíquicos”. En El Libro, Tomo I, 1908.

------. "La Escuela y la Revolución". En Revista Socialista Internacional, 15 de diciembre, número I, año I, 9 a 20, 1909a.

------. "Liga Internacional para la educación racional de la Infancia" y en el mismo número, "Congreso popular de Educación", "Internacionalismo escolar" y "Las universidades populares de Noruega", RSI, año I, n 3, 1909 b, 15/2.

-----. “La Comuna y la Educación”. RSI, año I, no 4, 15-18 de marzo de 1909, “La educación racionalista", Año I, tomo II, no 1, 1909 c, 15/ 6.

-----. "La educación racionalista". En Revista Socialista Internacional, Año I, tomo II, $\mathrm{n}^{\mathrm{o}} 1,5$ y 6,1909 d, 15/6.

. "La sociedad Luz, universidad Popular". Año I, Tomo II, 105-107, 1909e, 14 y $15 / 7$.

“La educación laica y la moral”. RSI, año I, no 4, 1909 f, 15 / 9.

-----. "La educación racionalista”, Año I, tomo II, nº 1, 1909 g, 15/9.

-----. "Feminismo e intelectualismo". En Humanidad Nueva, año 2, Tomo 3, nº 1 y 2, 1910a, 10/1.

-----. "La escuela laica. Sebastián Faure y Gustavo Hervé”. En Humanidad Nueva, año 2, tomo 3, n' 3, 1910b, 18/3.

. "La moral de la naturaleza". En Humanidad Nueva, año 2, Tomo 3, n 4, 1910c, $15 / 4$.

."Liga Nacional de Maestros". En Humanidad Nueva, Tomo III, nº 8, 1910d, $29 / 8$.

"Cómo se transforma el hogar". En Humanidad Nueva, Tomo III, n 10, 1910e, $13 / 10$.

------. La función endócrina del ovario. Tesis de grado, 57, 1914.

------. "El movimiento feminista en la República Argentina". Año I, no 1, 1919, $10 / 5$.

"Impresiones de viaje de la Dra. A. Moreau. Carta al Sr. A. Dóbrenky". año I, $n^{\circ}$ 9, 1920a, 10/1.

."El ensayo de voto municipal femenino". Año II, nº 19, 1920b, 10/11.

-----. “La acción social de la mujer estadounidense”. Año II, n 13. 1920 c, 10/5

-----. “Una carta y una respuesta”. Año II, no 15, 1920 d, 10/7.

-----. "Congreso Internacional de Médicos". Informe presentado al Ministerio de Justicia e Instrucción Pública del Congreso Internacional de Médicos, NY15/9/1922. En La Semana Médica, Tomo 27, 1922, 276-279

-----. El socialismo y la mujer. En La Vanguardia, Buenos Aires, 1933.

-----. "Conferencia Nacional de Mujeres Socialistas". LVF, primera plana, 1947, $3 / 6$.

-----. ¿Qué es un partido político? Buenos Aires: Talleres El Sol, 1952. 
------. La juventud argentina y el Partido Socialista. Buenos Aires: Ediciones Populares Argentinas, 1953.

-----. “¿Suspender los trámites de divorcio?”. En Ciudadana, n 2, 5, abril de 1956a.

-----. "El deber de la hora". En Ciudadana, n 6, 1956 b, 22 y 23.

-----. "Demencia paranoide". En Informe Médico Legal, s / d a.

-----. "Congreso Femenino Internacional". En Humanidad Nueva, Tomo 3, nº 6 y 7, 530 (a partir de convertirse en $H N$, la revista deja de precisar la fecha de publicación), s/d b.

Moreau, Alicia y Nerio Rojas. "Hemólisis experimental”. En Archivos Médicos, T. VII, 647, 1909.

Moreau, Alicia y Pedro Fernández. "Estudio experimental de la atención dinámica en el niño". En Trabajos de psicología normal y patológica. Horacio G. Piñero, Tomo I, 1905-1910, 1910.

Navarro, Marysa. Evita. Buenos Aires: Planeta, 1994.

Ramacciotti, Karina. “El Museo Social Argentino y el Primer Congreso de Población de 1940". En Sociohistórica, Cuadernos del Centro de Investigaciones Sociohistórica, No 13 y 14, 2003.

Ramacciotti, Karina y Adriana Valobra. "Modernas esculapios: acción política e inserción profesional, 1900-1950". En Género y Ciencia: hombres, mujeres e investigación científica en América Latina, siglos XVIII-XX. María Eugenia Scarzanella y Jacinto Lizette(comp). Colección Estudios AHILA de Historia Latinoamericana, volumen 8, Asociación de Historiadores Latinoamericanistas Europeos, Madrid/Frankfurt, 2011.

Rey, Ana Lía. “Revistas feministas publicadas por mujeres socialistas. Buenos Aires 1900-1956". En VI Encuentro de historiadores de la prensa y el periodismo en Iberoamérica San José, Costa Rica, 2010.

Román, Selene. "Consideraciones para el estudio de género y comunicación en publicaciones feministas de principios del Siglo XX: revista Vida Femenina (1933)". En X Jornadas de Historia de las Mujeres V Congreso Iberoamericano de Género, Luján, 2010.

Talak, Ana María. “Progreso, degeneración y darwinismo en la primera psicología argentina. 1900-1920". En Derivas de Darwin. Cultura y política en clave biológica. Vallejo, Gustavo y Marisa Miranda. Buenos Aires: Siglo XXI, Ed. Iberoamericana, 2010.

Terán, Oscar. Nuestros años sesentas: La formación de la nueva izquierda intelectual argentina 1956-1966. Buenos Aires: Ed. El cielo por asalto, 1993.

-----. Vida intelectual en el Buenos Aires fin de siglo (1880-1910). Derivas de la cultura científica. Buenos Aires: FCE, 2000.

Vallejo, Gustavo. "La casa moderna y los derechos políticos de la mujer”. En Sociohistórica. Cuadernos del CISH. La Plata, N 9, 2001.

Valobra, Adriana. Del hogar a las urnas. Recorridos de la ciudadanía política femenina. Argentina, 1946-1955. Rosario: Prohistoria, 2010.

Vezzetti, Hugo. La locura en la Argentina. Buenos Aires: Paidós, 1985. 
